\title{
LA CONSTRUCCIÓN DE PUENTES COLGANTES \\ EN COLOMBIA DURANTE EL SIGLO XIX: ENTRE LA TRADICIÓN Y LA INNOVACIÓN*
}

Jorge Galindo Díaz***

Puente sobre el río Suaza en el Tolima. Del Ingeniero E. G. Barney (grabado de Vargas) Fuente: Papel períodico ilustrado, Bogotá, vol.4 No. 84. febrero 5 de 1885 .

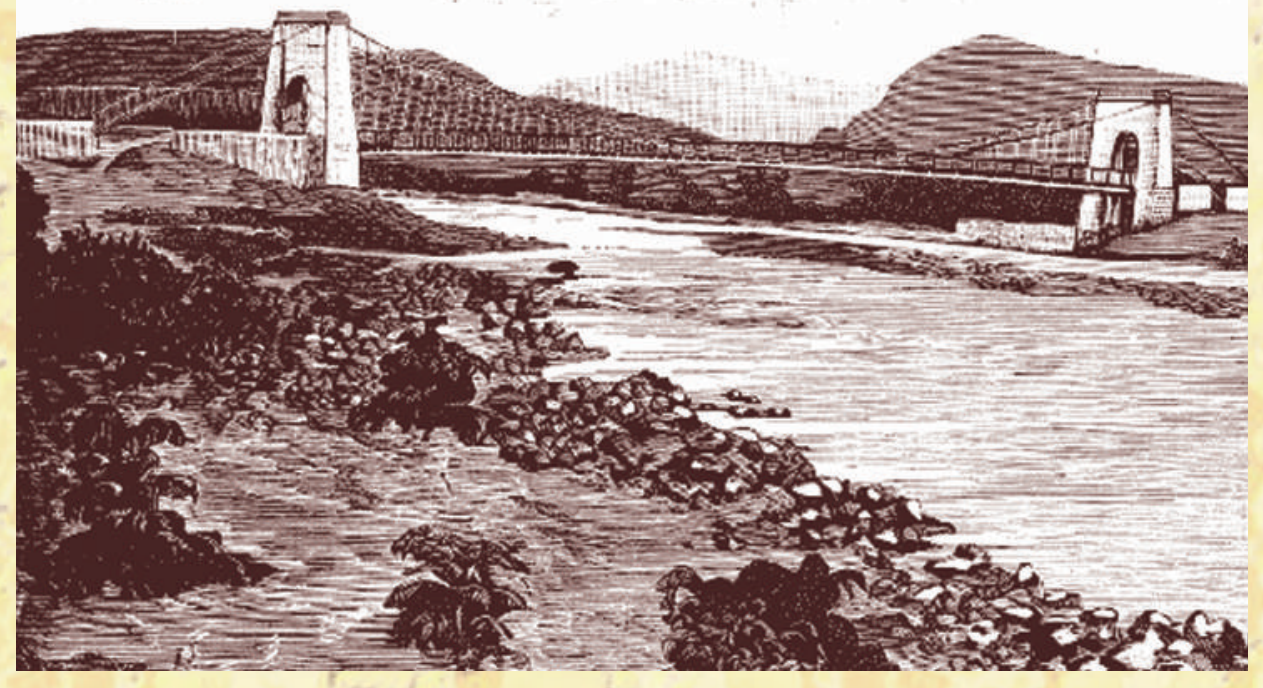

Artículo de investigación científica y desarrollo tecnológico. Resultado parcial de la investigación Técnica y poder. Historia de las obras públicas en el Valle del Cauca, financiada por la Dirección de Investigaciones - DIMA de la Universidad Nacional de Colombia, sede Manizales.

\section{RESUMEN}

Este artículo explora la manera en que la construcción de puentes en Colombia durante la segunda mitad del siglo XIX se llevó a cabo mediante una mezcla de tradiciones artesanales y sistemas industrializados. Las primeras, tienen sus raíces en tiempos prehispánicos e involucran el trenzado de fibras vegetales utilizadas en la construcción de puentes colgantes. Por su parte, los sistemas industrializados fueron poco a poco introduciéndose al país de la mano de fabricantes extranjeros, que vendían sus estructuras metálicas por todo el mundo a través de catálogos y buscaban siempre adaptarse a las más complejas condiciones locales. A través del estudio de la ingeniería colombiana de este período y sus logros constructivos, se intenta demostrar esta condición.

\section{PALABRAS CLAVE}

Puentes colgantes, tradición constructiva, innoyación tecnológica. 


\section{HANGING BRIDGE CONSTRUCTION IN COLOMBIA DURING THE NINE TEENTH CENTURY: BETWEEN TRADITION AND INNOVATION}

Jorge Galindo Díaz
Arquitecto de la Universidad del Valle y Doctor en Arquitectura de la Escuela Superior de Arquitectura de Barcelona. Profesor Titular de la Escuela de Arquitectura y Urbanismo de la Universidad Nacional de Colombia, sede Manizales.

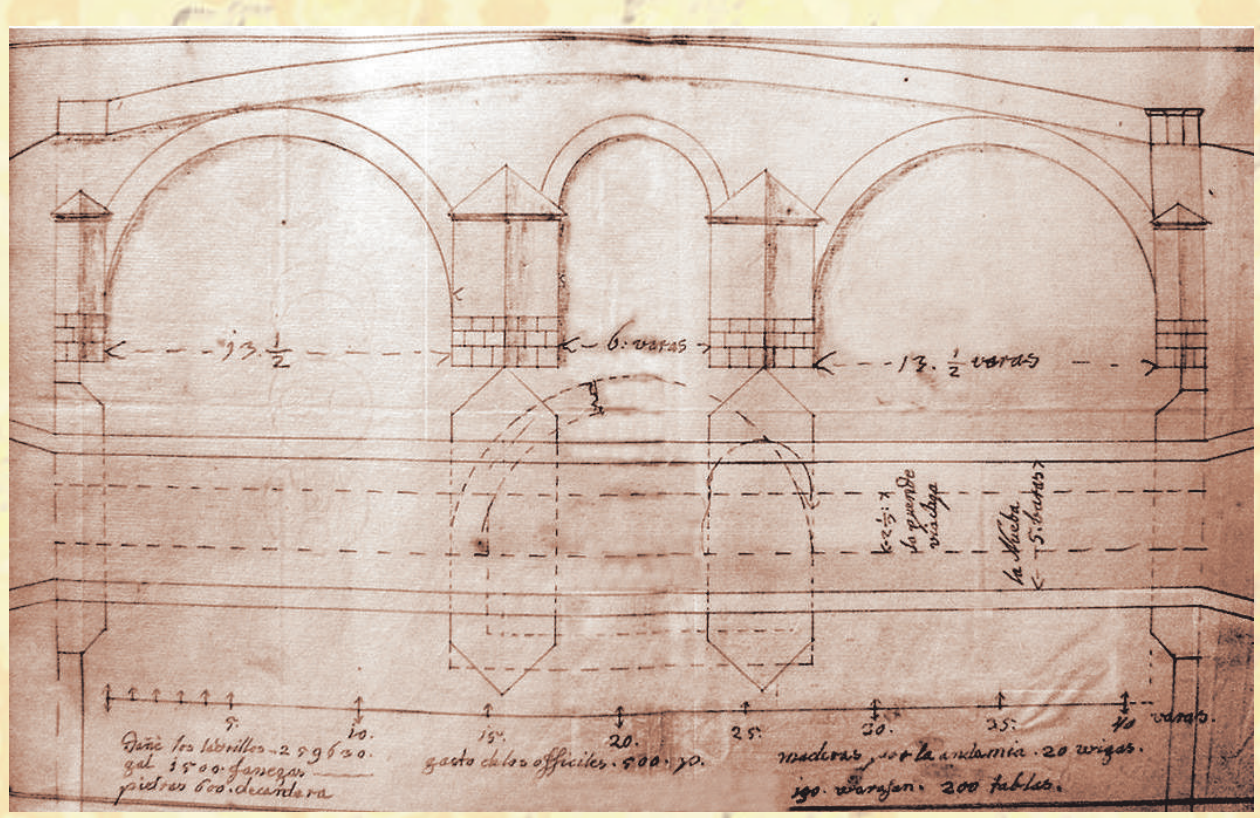

\section{ABSTRACT}

In this presentation, we explore how the construction of bridges in Colombia during the second half of the 19th century was conducted using a mix of artisan tradition and system from the era of industrialization. The first of these date back to Pre-historic times and involve the braiding of vegetable fibers used in the construction of hanging bridges; at the same time, standardized systems were established by foreign manufacturing entities, which sold their metallic structures around the world through catalogs seeking to adapt to the most complex local conditions. Though the study of a Colombian engineer from the period and his construction work, we aim to demonstrate this condition.

\section{KEY WORDS}

Suspension bridges; construction tradition, technological innovation
Proyecto de Simón Schenherr de un puente con arcos de ladrillo sobre el río Cauca en Popayán. Fuente: Archivo Histórico del Cauca. Cabildo. 1753. 


\section{INTRODUCCIÓN}

Es un hecho reconocido que la figura del ingeniero como la conocemos hoy en día, nació en los siglos XVII y XVIII al compás de la ciencia moderna. Varios autores (Vérin, 1993 y Picón, 1988, principalmente) han explicado la manera en que esta nueva profesión surgió a partir de las necesidades sociales de los nuevos Estados europeos que demandaban entonces no sólo de una regularización de prácticas sino también de una fundamentación y búsqueda de legitimación matemática de muchas actividades tradicionales, todo ello con el fin de superar las de carácter puramente artesanal.

En Colombia, un fenómeno similar se reprodujo a lo largo de la segunda mitad del siglo XIX, caracterizado tanto por la institucionalización de la tecnología y los sistemas tecnológicos, como por el ascenso profesional del ingeniero, situado en un lugar intermedio entre el Estado y la empresa privada, sometido, por una parte, a las fuerzas de la necesidad de la estandarización y, por otro, a las presiones del beneficio económico. Su génesis como elite intelectual y como gremio ha sido estudiada en el contexto local (Safford, 1976 y Obregón, 1992), quienes hacen énfasis en el esfuerzo por la cualificación de sus actividades y en las disputas libradas contra el intrusismo profesional.

Sin embargo, en Colombia, como en otras naciones americanas, la ingeniería civil tuvo algunos precedentes importantes desarrollados dentro de las civilizaciones precolombinas, los cuales indudablemente forjaron una tradición de artesanos que irían extinguiéndose con el pasar del tiempo, lo que en buena medida puede explicarse por el desdén que las prácticas europeas manifestaron hacia casi todo lo que no fuera parte de su misión civilizadora.

La primera parte de este artículo destacará algunos logros concernientes a la construcción de puentes colgantes en suelo americano llevados a cabo por mano de obra indígena. La segunda parte tratará de la construcción de puentes colgantes durante la segunda mitad del siglo XIX bajo la dirección de ingenieros locales y extranjeros, quienes fueron capaces de mezclar algunas prácticas artesanales con innovaciones producidas por el mundo industrializado.

\section{PUENTES COLGANTES EN AMÉRICA PREHISPÁNICA}

En los extensos territorios americanos la construcción primitiva de puentes colgantes tuvo un origen incierto que es además imposible de datar. La falta de escritos entre las comunidades indígenas prehispánicas y la dificultad para establecer fechas de sus logros constructivos obliga a recurrir a documentos redactados por los propios españoles durante el período de conquista. Se sabe así que en algunos casos, los españoles se sorprendieron por las técnicas de construcción y por las dimensiones empleadas en el desarrollo de este tipo de estructuras. Por ejemplo, un sacerdote jesuita del siglo XVIII describe de la siguiente manera la existencia de puentes suspendidos en México durante esa época (Clavigero, 1826: 352):

El puente más singular de los usados en aquellos países era el que los españoles llamaron hamaca. Era un tejido de cuerdas naturales de cierto árbol más flexible que el mimbre, pero más grueso y fuerte, llamado en América bejuco, cuyas extremidades colgaban de dos árboles de orillas opuestas, quedando el tejido colgando en medio, a guisa de columpio. Todavía se ven puentes de esta especie en algunos ríos. 
Para entonces, ya otros autores del siglo XVII habían hecho referencia a puentes suspendidos como el del río Apurímac, en Perú, que generaba un fuerte temor entre aquellos que tenían que atravesarlo (Garcilaso, 1984: 108):

La [puente] de Apurímac, que es la más larga de todas, tendrá doscientos pasos de largo. No la medí, más tanteándola en España con muchos que la han pasado le dan este largo, y antes más que menos. Muchos españoles vi que no se apeaban para la pasar y algunos la pasaban corriendo a caballo, por mostrar menos temor, que no deja de tener algo de temeridad.

Casos como este fueron registrados aún hasta la mitad del siglo XIX a través de ilustraciones que han sido preservadas como acompañamiento de crónicas de expediciones extranjeras a lo profundo de los territorios andinos, tal como se aprecia en la Figura I.

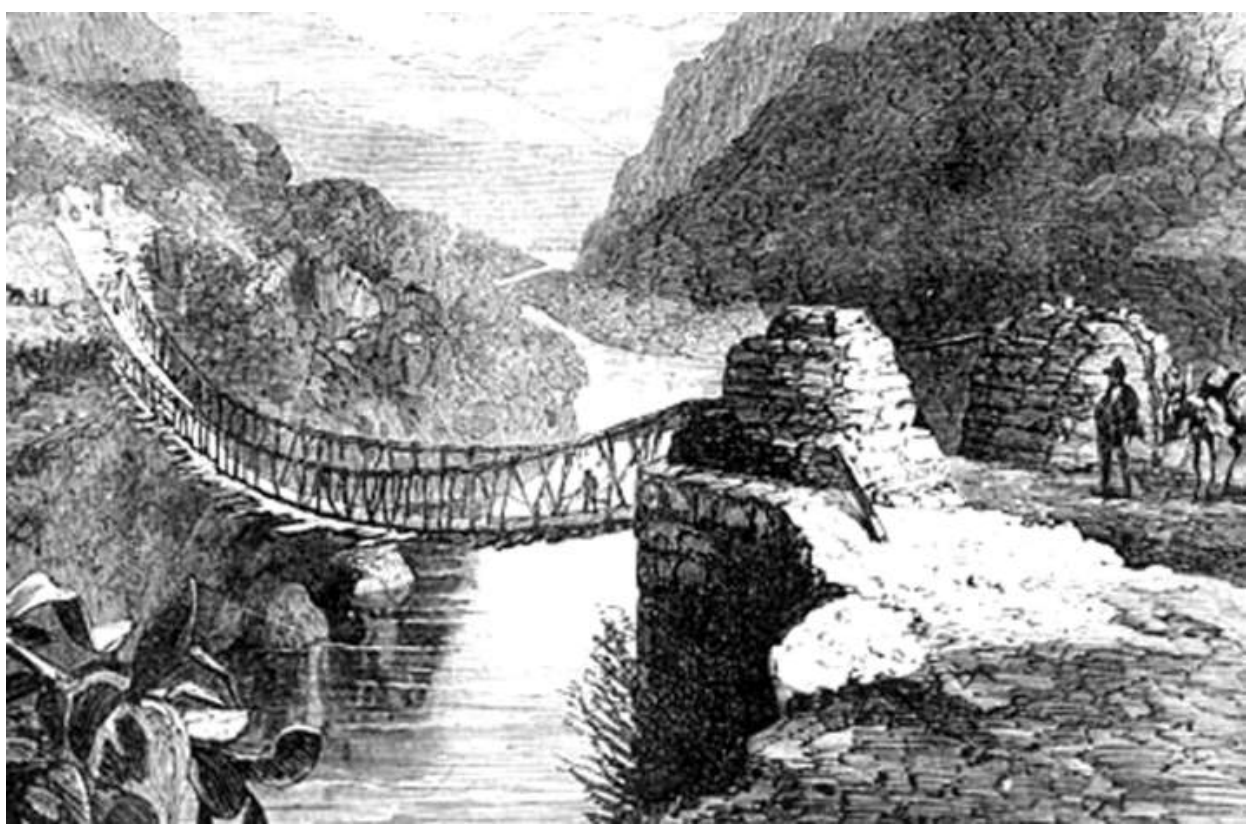

Es fácil deducir que en estos puentes, como en los que se construirían años más tarde con métodos industrializados, la elección de un sitio apropiado era el primer paso en el proceso de construcción: debido a su forma geométrica, debían quedar protegidos de las corrientes de los ríos, ubicándose en la mayoría de los casos donde el cauce fuese estrecho y permitiendo siempre que la distancia libre entre los extremos fuese a la vez la más corta posible.

Existen pruebas, gracias a muchas citas similares a las mencionadas anteriormente, de que los materiales usados en la fabricación de los cables no eran más que fibras vegetales trenzadas las cuales conformaban cuerdas muy gruesas resistentes a la tracción. Las cuerdas, a su vez, se acompañaban de piezas de madera atadas entre sí para dar forma al tablero o piso aunque también estas últimas podían usarse como cuñas allí donde las cuerdas se ataban por sus extremos a bloques naturales de piedra que servían de apoyos. En cualquier caso, la corta vida de los materiales demandaba un constante trabajo de mantenimiento sin que se llegase a contar con una fórmula única para la fabricación de los componentes, dando pie a técnicas que podían variar de un lugar a otro.
Figura I. Puente de fibras vegetales sobre el río Pampas (Perú), en una crónica de 1865. Fuente: (Squier, 1877: 558) 
Lo que sí queda claro es que a lo largo y ancho del territorio americano llegó a existir un delicado trabajo hecho a mano, el cual abarcó desde el secado de fibras extraídas de diferentes variedades de plantas hasta el difícil trabajo de atarlas, unirlas y tejerlas para lograr al fin una pieza de gran longitud y alta resistencia. Así, fue posible para muchas de las comunidades indígenas, viajar a través de una compleja geografía, sobre una vasta red de senderos y salvar difíciles obstáculos naturales.

Al mismo tiempo, entre los siglos $\mathrm{XVl}$ y $\mathrm{XIX}$ los españoles empezaron a construir numerosos puentes de ladrillo y piedra mediante estructuras de arcos (complejos en su proceso de elaboración y pesados en su estructura), que no sólo serían costosos (dada la fuerza de trabajo especializado y la dificultad en la consecución de sus materiales) sino también ineficientes en virtud de sus luces cortas e inestables frente al cauce de los ríos. Sería entonces necesario esperar la llegada de las nuevas técnicas de construcción metálica, utilizadas en América en las postreras décadas del siglo XIX, para ver de nuevo puentes colgantes que cruzaran las montañas de los Andes.

\section{PUENTES EN COLOMBIA DURANTE EL SIGLO XIX}

Desde el comienzo de la colonización española y hasta el término de la primera mitad del siglo XIX, la construcción de puentes en Colombia se desarrolló en torno a dos sistemas: bóvedas de ladrillo y celosías de madera. Así, cuando en 1753 se necesitaba construir un puente sobre el río Cauca, en inmediaciones de Popayán, el sacerdote alemán Simón Schenherr presentó dos proyectos, uno en cada sistema (Figuras 2 y 3 ), incluyó un análisis de las ventajas y desventajas que ambos presentaban. Finalmente, ninguno de los dos proyectos se llevó a cabo por razones que todavía desconocemos.

Figura 2. Proyecto de Simón Schenherr de un puente de madera sobre el río Cauca, en Popayán. Fuente: Archivo Histórico del Cauca,

Cabildo, 1753.

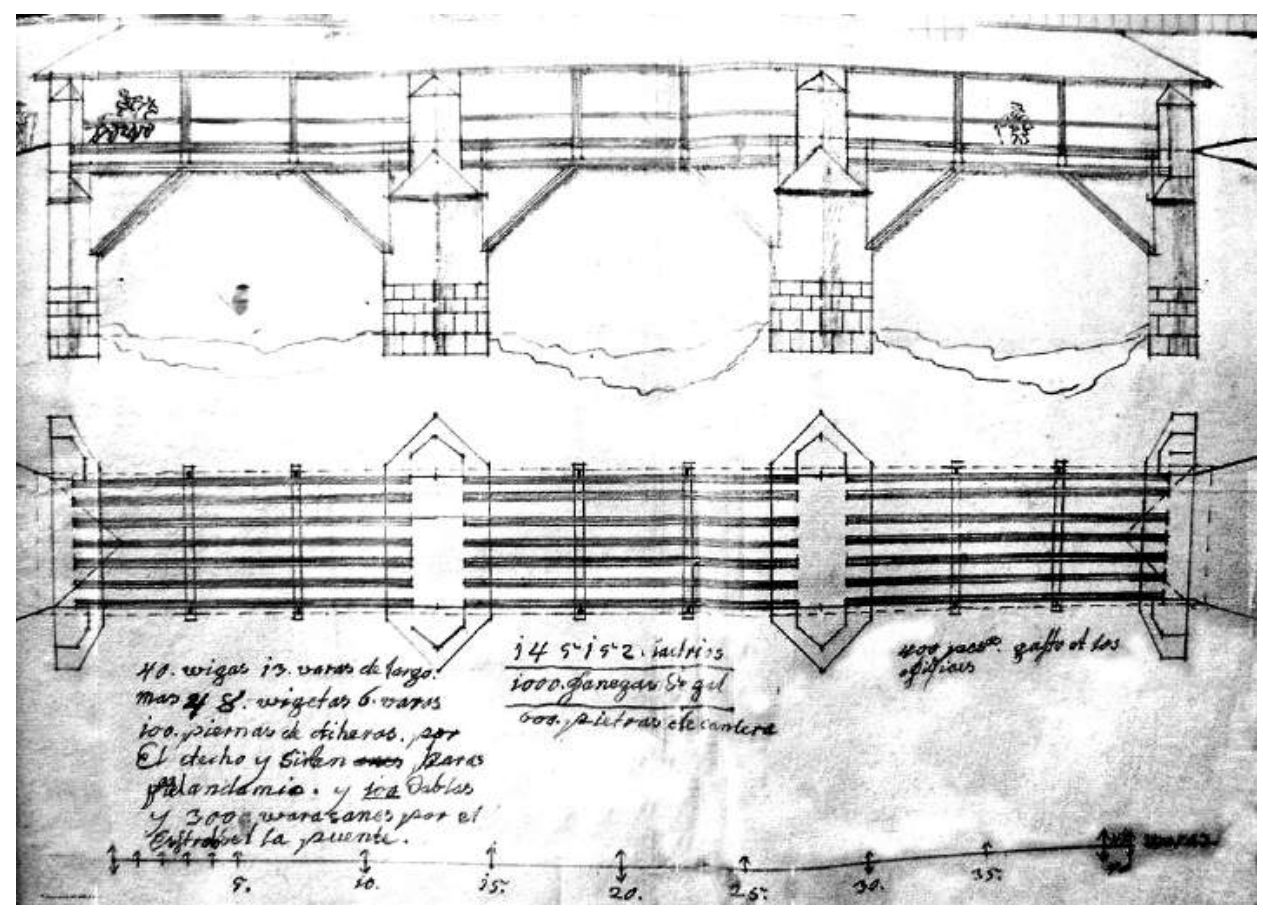




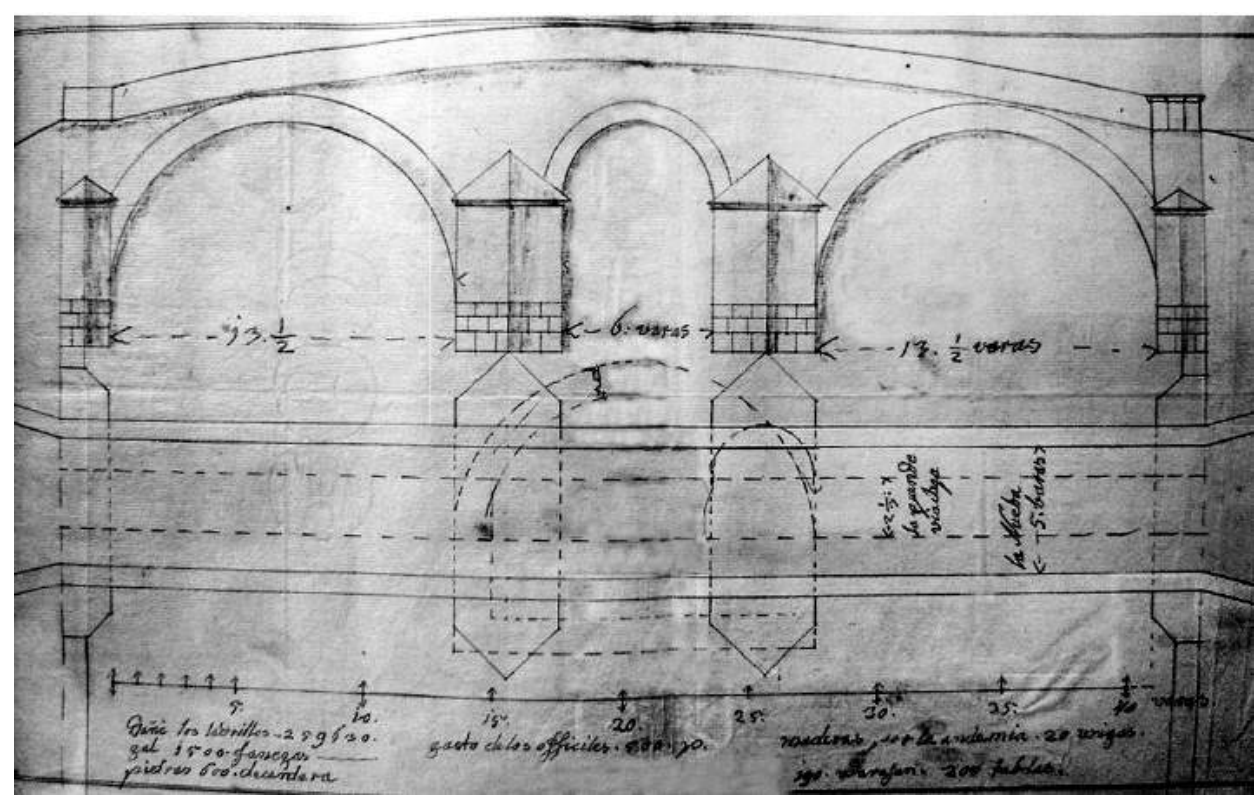

El sistema de puentes de arco de ladrillo fue ampliamente usado a tal punto que en la región del sur occidente del país -entre 1865 y I895-, se construyeron por lo menos 40 de ellos (Galindo \& Paredes, 2008), muchos de los cuales fueron diseñados y supervisados por sacerdotes y pseudo arquitectos de origen europeo, cuya plena aceptación fue posible gracias a su buena durabilidad y al desarrollo de técnicos locales que progresivamente se fueron cualificando hasta llegar a participar en numerosas construcciones civiles y religiosas en toda la región.

Los puentes de madera, por su parte, fueron menos deseados, aunque más baratos y fáciles de hacer por parte de los carpinteros locales, como en el caso del artesano Valentín Cadavid, aclamado por ser el más hábil constructor de puentes bajo la forma del llamado sistema antioqueño que no era más que una estructura rústica de vigas de madera que formaban entramados paralelos, con cubierta a dos aguas y apoyos simples sobre muros de fábrica de piedra o ladrillo. Se trataba de estructuras poco durables que requerían continuas y permanentes reparaciones en medio de senderos difíciles, donde las distancias eran largas y el dinero escaso. Este sistema estaría en uso hasta bien entrado el siglo $\mathrm{XX}$ y fue ampliamente usado en diferentes zonas de la región central de Colombia, como lo atestiguan planos y memorias que aún se conservan en el Archivo Histórico Nacional, en Bogotá (Figura 4).

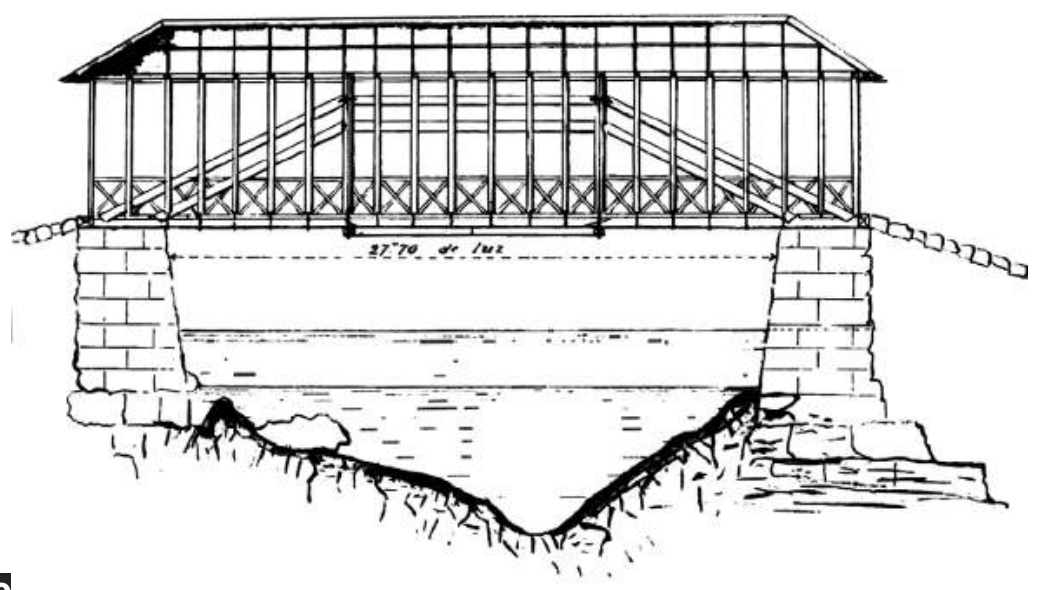

Figura 3. Proyecto de Simón Schenherr de un puente de arcos de ladrillo sobre el río Cauca, en Popayán Fuente: Archivo Histórico del Cauca, Cabildo, 1753

Figura 4. Puente según el sistema antioqueño diseñado por Felipe Escobar para salvar el puente sobre el río Guarinocito (Caldas) en I887 Fuente: Archivo General de la Nación, Bogotá, Sección República, Fondo Ministerio de OOPP, t. 1483, f. 408. 
Por su parte, la construcción de puentes metálicos en Colombia se vería seriamente retrasada por la ausencia de materias primas: aún en la segunda mitad del siglo XIX el hierro era un material escaso y caro, por lo que las armaduras debían ser compradas casi por entero a casas fabricantes de Londres, Nueva York o Bremen. A pesar de ello, las noticias llegadas de Europa y Norteamérica acerca de los nuevos logros de la ingeniería en torno a la construcción metálica y el fuerte impacto que tuvo en la sociedad local el arribo de la empresa del ferrocarril, serían motivos suficientes para que empresarios nacionales invirtieran esfuerzos y economías en la compra y montaje de puentes con luces antes no imaginadas: el del río Suaza, en el Tolima, a cargo del ingeniero E.G. Barney lograba los 87,78 m de luz en 1884 y bien puede considerarse pionero en este ámbito (Figura 5), seguido del puente que a cargo de José María Villa se levantó sobre el río Cauca en la población de Santafé de Antioquia en 1891 , de casi 300 m de luz.

Figura 5. Puente sobre el río Suaza, en el Tolima, del ingeniero E.G. Barney (grabado de Vargas). Fuente: Papel periódico ilustrado, Bogotá, vol. 4, No. 84, febrero 5 de 1885 .

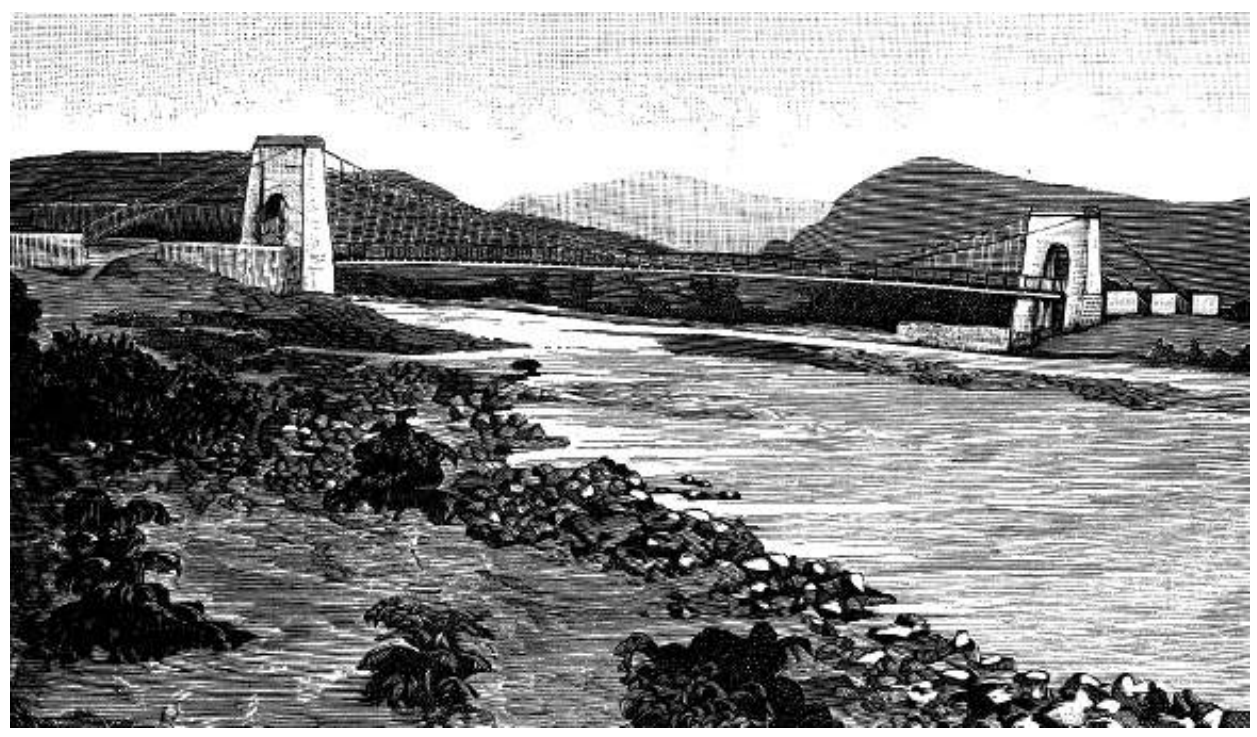

Y aunque en muchos casos podría resumirse la experiencia tecnológica introducida en Colombia a través de la construcción de puentes colgantes por toda su geografía, vale la pena para las intenciones de este artículo detenerse a explicar un reducido número de casos individuales que no son muy lejanos a la norma general experimentada por los nóveles ingenieros al servicio del país.

\section{CENÓN CAICEDO, INGENIERO AUTODIDACTA}

El papel jugado por Cenón Caicedo ( I827-1900) brinda un ejemplo interesante de una figura prototípica del ingeniero colombiano durante la segunda mitad del siglo XIX. Se sabe que nació en Cali y realizó sus estudios en el recién creado Colegio de Santa Librada, donde con seguridad recibió clases de literatura, matemáticas y filosofía, aunque de mayor se decantaría por el ejercicio de la contabilidad. Ocupó numerosos cargos, entre los cuales figuran el de Colector de Rentas del Cantón de Cali (1850), Administrador de Correos de la provincia de Buenaventura (185 I-1853), Tesorero General de Guerra del Ejército, Administrador de Hacienda de la provincia de Cali, miembro de la Junta Directiva del Camino de Cali a Palmira ( 187I- 1876) y contador al servicio del ingeniero cubano Francisco Javier Cisneros durante la construcción de algunos tramos ferroviarios entre Buenaventura y Cali. Ya como empresario independiente se dedicó al estudio de las construcciones y maquinarias 
recibió el privilegio para montar en Cali la primera fábrica de materiales de construcción (ladrillo y adobes principalmente) domiciliada en el sector de Santa Mónica. Al final de sus años se consagró a la construcción de puentes metálicos colgantes. Su carácter de autodidacta fue bien destacado mientras ejerció su actividad como técnico y recientemente refrendado por Safford (1976: 330) y Poveda (1993: 152/II).

Su primera incursión dentro del campo de la construcción de puentes colgantes tuvo lugar en I 883 cuando fue comisionado por el gobierno provincial para construir un puente de $34,26 \mathrm{~m}$ de luz sobre el río Palo, destinado a reemplazar una estructura de madera construida en 1879 y arrasada por una creciente del río un año después. La obra debería quedar garantizada en su solidez por un espacio de 20 años y el plazo para su construcción era de 6 meses, a un costo global de $\$ 9.900$ pesos. Se deduce que la estructura metálica era importada puesto que en el contrato (publicado en Registro Oficial del Cauca, No I72, febrero I I de I882) se hacía referencia a que el dinero del anticipo lo invertiría Caicedo en el transporte de las piezas desde el puerto marítimo de Buenaventura.

Sin embargo, los trabajos no serían fáciles. Un año después de encargadas las obras, y mediante Decreto, se dispuso por parte de las autoridades del Estado una visita de inspección al puente:

Teniendo en consideración que al construir el puente de hierro sobre el río Palo, por ser la primera obra de esta clase que se construye en el país, se le han presentado al contratista algunas dificultades para concluirla dentro del término fijado en el respectivo contrato, dificultades que no podrán vencerse sin la presencia del Gobierno en el lugar de la obra ... Decreta: Art. $1^{\circ}$. Señálase el día 19 del presente para practicar la visita de inspección en la obra del puente de hierro sobre el río Palo (En Registro Oficial del Cauca, No. 24I, abril 2 I de 1883).

Para esta visita el Gobierno decidió contratar la opinión de un experto, el ingeniero italiano Gaspare Mazza, residente en Cali y cuyo informe, fechado el mayo 26 de 1883 contenía el siguiente concepto:

El puente sobre el río Palo que se está construyendo es de buen tipo, y se ve que los detalles fueron estudiados por persona práctica en el arte.

Los materiales de hierro fueron trabajados en la fábrica con la precisión que se requiere. Los de madera que yo ví, son también de buena calidad y cortados con precisión. Los ladrillos en acopio son de buena calidad, y bien hechos, y la parte aparente de la mampostería la encontré bien hecha también (...)

Haciendo el cálculo de la resistencia de la cadena de fierro que sostiene el puente, se encuentra que el hierro queda bajo el esfuerzo mínimo de cerca de 4,50 por metro cuadrado, cuando el puente está sin carga, y bajo carga de 130 kilogramos por metro cuadrado de superficie, el esfuerzo queda de 9 kilogramos para medio metro cuadrado, lo que puede ser aún tolerable para un esfuerzo accidental y no permanente (...)

El esfuerzo que deben soportar los tirantes verticales es menor en los dos casos, y el puente podrá resistir al pasaje de carros comunes (En Registro Oficial del Cauca, No 250, junio 15 de 1883). 
Figura 6. Puente colgante sobre el río Tuluá a la entrada de la población por el lado norte. Fuente: Cromos, Bogotá, No. II, marzo 26 de 1916; p. 173.
Frente al temor y la duda que brindaba la nueva tipología constructiva, el análisis matemático parecía dar la razón a la obra y a su constructor. Además, fue una práctica común para este tipo de proyectos la clara división de dos actividades diferentes: la construcción de las obras de albañilería (cimientos, bases de sujeción y todo tipo de obras accesorias) y el ensamblaje propiamente dicho de la estructura metálica importada.

Finalmente, el puente puesto en servicio en 1884 , causó la admiración de los habitantes de la región y llevó a que el ingeniero Caicedo fuese casi inmediatamente comisionado en la delicada tarea de levantar nuevos puentes a fin de modernizar los caminos de una región que buscaba desesperadamente el transporte de sus productos agrícolas hacia el puerto marítimo de Buenaventura, punto intermedio en el transporte comercial entre Panamá y Lima.

Entre 1888 y 1889 Cenón Caicedo dirigió simultáneamente la construcción del puente colgante sobre el río Tuluá (Figura 6) y el puente sobre el río Amaime en un punto intermedio a las ciudades de Palmira y Buga. En ambos casos las estructuras metálicas fueron compradas a la casa de los hijos de John Roebling, en Trenton (New Jersey, USA).

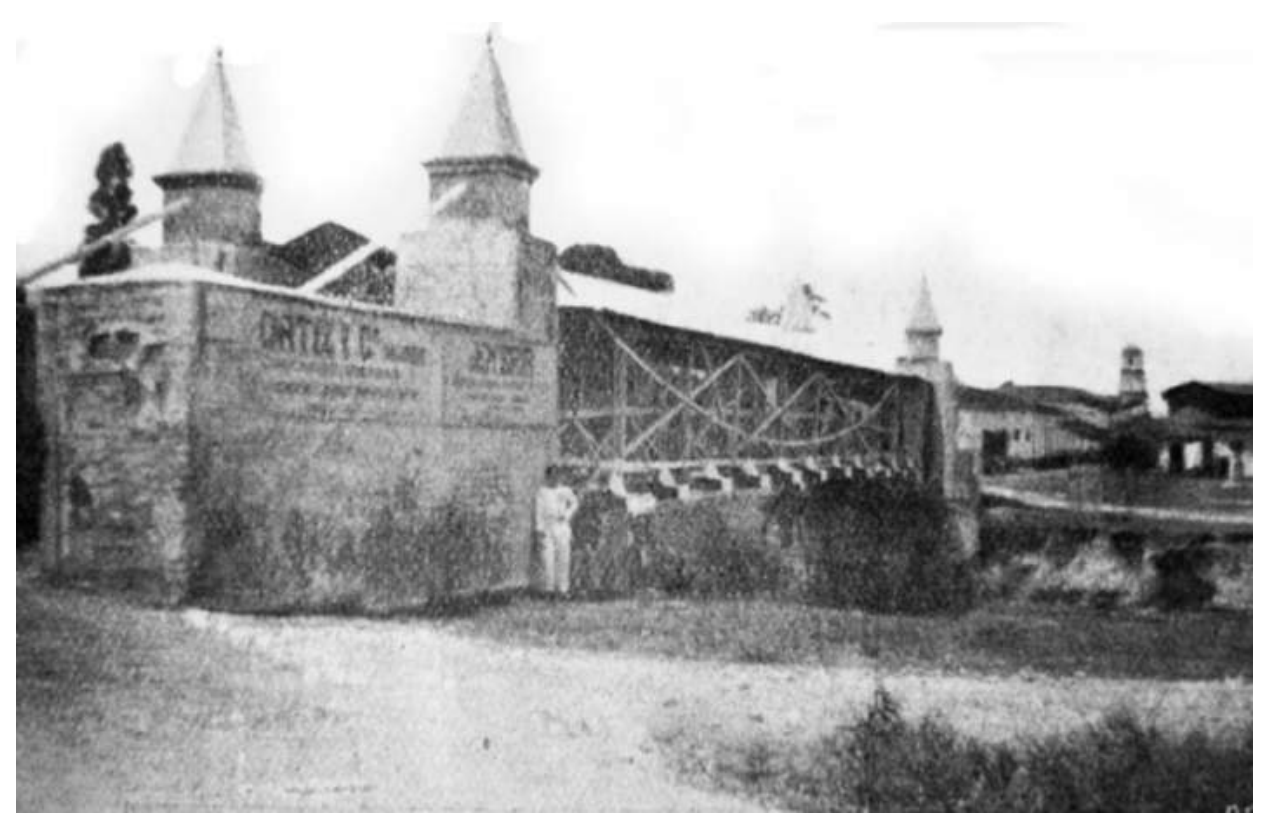

En 1890 Cenón Caicedo inició la construcción de otro puente metálico colgante sobre el río Amaime, pero esta vez en el llamado camino de La Torre, que desde la población de El Cerrito cruzaba el río Cauca en un punto cercano a Yumbo y desde allí llegaba por el norte a Cali. Sus características constructivas han quedado consignadas en el acta de entrega de la obra, firmada un año más tarde:

Las pilastras tienen, de un extremo á otro, una extensión de cinco metros cincuenta y cuatro centímetros, y la entrada ó sea la distancia comprendida entre una pilastra y otra, es de dos metros cuarenta y cinco centímetros.

Altura de las torres, cinco metros siete centímetros, fuera del asiento de las cadenas; la extensión del puente, obra de madera, es de treinta y ocho metros 
noventa y cinco centímetros. La extensión de los estribos es de ocho metros cuarenta y cuatro centímetros, y el espesor es de cinco metros cincuenta y cinco centímetros. La altura del puente sobre el nivel de las aguas es de cuatro metros veintiséis centímetros ...

Los estribos están separados de la orilla de las aguas, el del Norte que queda en la playa, once metros cincuenta centímetros y el del Sur, cinco metros noventa y cinco centímetros, estando en este lado la peña del río (En Registro Oficial del Cauca, sn, septiembre 4 de I89I).

Sin embargo, de todos sus puentes, el del paso de Aganche sobre el río Ovejas fue el que mayor admiración despertó, tal y como lo registra una nota especial publicada en Anales de Ingeniería que incluye la trascripción del acta de recibo de la obra en la cual se describen sus aspectos constructivos:

- Fundación de los cimientos de los estribos: (...) la de la orilla derecha á una profundidad de 3 metros bajo el nivel ordinario del agua, sobre terreno incomprensible y tenaz, con piedras grandes del río, dispuestas en capas horizontales y calzadas con hormigón, hasta la altura de 2 metros; de allí para arriba se construyó con calicanto hasta la altura de 3 metros sobre el mismo nivel (...)

- Estribos: Estos se han levantado sobre una base de $8 \mathrm{~m} .40$ de largo por $5 \mathrm{~m} .80$ de frente, con sus correspondientes zarpas, y sobre el cual se levantaron los muros en que van aseguradas las anclas que sostienen las cadenas, hasta una altura de $4 \mathrm{~m} .45$ por $8 \mathrm{~m} .23$ de largo y un espesor de $\mathrm{Im} .56$. En los paramentos que dan frente al río, y sobre los muros descritos, se han levantado las cuatro torres que soportan los galápagos colocados sobre piedras de cantera talladas. Estas torres tienen una base cuadrada de $1 \mathrm{~m} .56$ y una altura de $2 \mathrm{~m}$.

- Materiales de hierro: La catenaria de cada lado está formada por trece pares de eslabones de acero de $2 \mathrm{~m} .55$ de largo, $0 \mathrm{~m} .90$ de ancho y $0 \mathrm{~m} .016$ de espesor, unidos en sus extremidades por tornillos de acero de $0 \mathrm{~m} .038$ de diámetro, de los cuales están suspendidos los colgantes de hierro apareados, de longitudes decrecientes de los estribos al centro y un diámetro de $0 \mathrm{~m} .02$ de espesor, los que soportan las doce vigas que sostienen el tablero del puente. El peso de estos materiales es de 358I kilogramos.

- Obras de madera: Esta está apoyada sobre las doce vigas colocadas de canto de que se habla en el punto anterior, y que tienen $5 \mathrm{~m} .48$ de largo, $0 \mathrm{~m} .20$ de ancho y $0 \mathrm{~m} .055$ de grueso, unidas una á otra por cruces de San Andrés, que sirven de contra vientos (...) (En Anales de Ingeniería, vol. V, No. 57, abril de I892; p. 277-278).

El puente tenía 30,48 m de longitud, y estaba situado a 100 m del paso público, pues en el punto elegido, el cauce del río apenas alcanzaba los $26 \mathrm{~m}$ de ancho y se recostaba sobre la orilla izquierda, la que además de ser capaz de resistir á la fuerza de la corriente, llegaba a una altura de más de $10 \mathrm{~m}$; la orilla opuesta, aun cuando mucho más baja, no alcanzaba a ser anegada por el río en sus grandes avenidas. $Y$ pese a las altas especificaciones del puente, lo que más interés causó entre quienes lo conocieron fue el uso de cable trenzado para telegrafía en la construcción de las curvas parabólicas.

En efecto, a causa de la pérdida de los cables originales de acero durante el transporte de las piezas desde el puerto de Buenaventura hasta el sitio de construcción, Caicedo se vio en la obligación de prescindir de las piezas importadas desde Trenton y echar mano de 
Figura 7. Lugares en donde Cenón Caicedo construyó puentes. Fuente: elaboración propia artesanos locales, quienes a la manera de sus ancestros indígenas, trenzaron manualmente trozos de cable de telégrafo, ajustándolas con anillos de cobre para ponerse así al servicio de la estructura.

No hay certeza de que Caicedo haya aplicado con anterioridad este mismo tipo de procedimiento en otros puentes construidos bajo su dirección; pero lo que sí es claro, en virtud de la evidencia documental de este caso, es que durante las últimas décadas del siglo XIX, la milenaria tradición artesanal se asoció con los sistemas industrializados recién llegados al país.

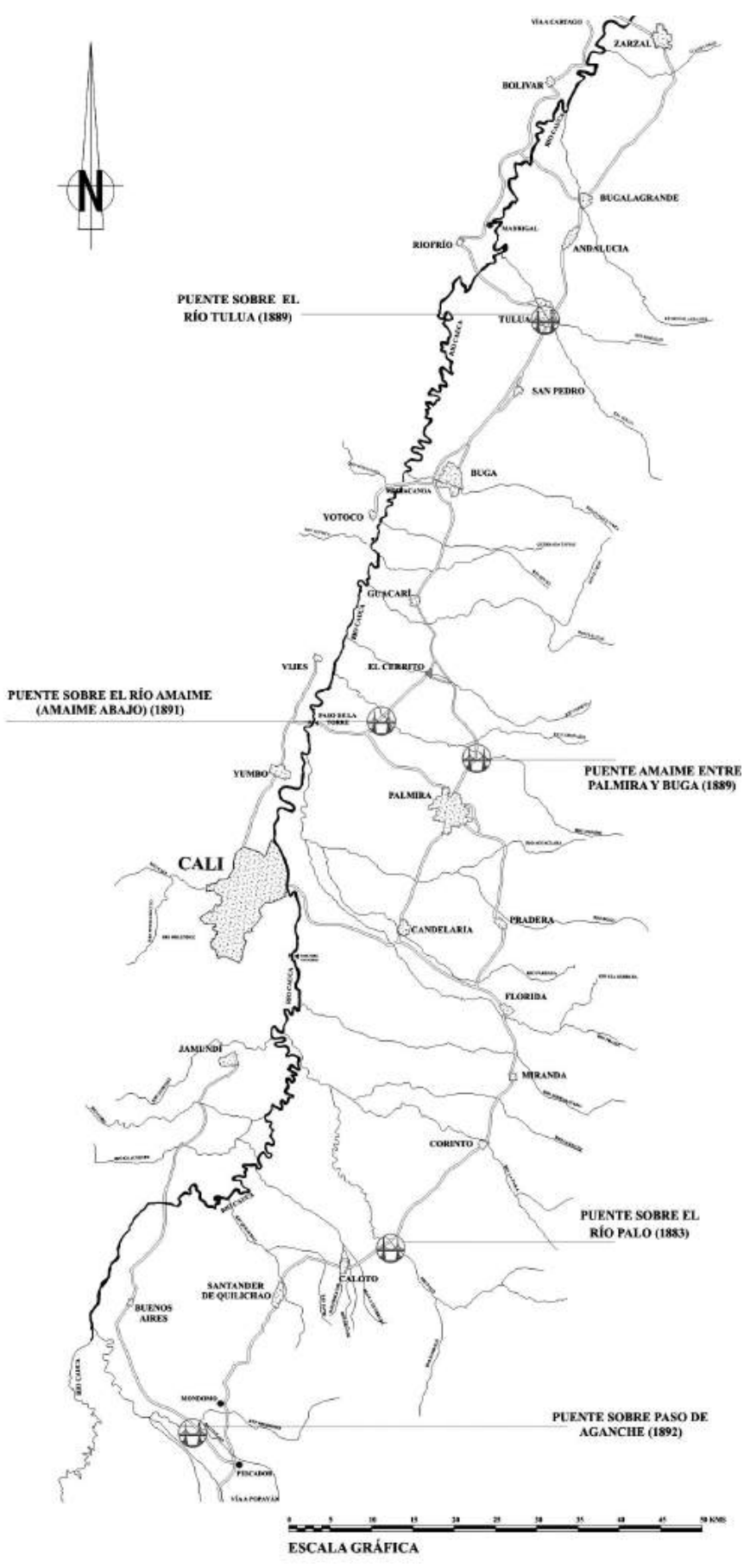


El ingeniero Cenón Caicedo falleció en 1900, dejando por lo menos seis puentes colgantes en operación por toda la región (Figura 7). Su figura se convirtió en un modelo para las siguientes generaciones, hasta que fue relegado al olvido al mismo tiempo que llegaban, poco a poco, estructuras de hormigón armado que sumían en la indiferencia a los puentes metálicos colgantes.

\section{CONCLUSIÓN}

El breve caso expuesto aquí permite identificar una mezcla de tradición e innovación utilizada por los ingenieros colombianos durante el advenimiento de su profesión en la segunda mitad del siglo XIX. Es posible pensar que ellos decidieron ser parte del mundo de la tecnología industrializada aunque las limitaciones impuestas por el tiempo y la distancia les obligaron en muchos casos a hacer uso de tradiciones artesanales propias de los habitantes de la región. Pese a ello, los conocimientos ancestrales empleados en la construcción de muchos puentes colgantes durante el período precolombino no fueron considerados parte de su utillaje cultural y, por el contrario, fueron relegados y olvidados pese a que en ocasiones, emergían como útiles y necesarios. El universo de la técnica parecía no ser consciente de las claras fronteras entre conocimientos dominantes y dominados.

\section{REFERENCIAS}

Clavigero, F.S. ( I 826): Historia antigua de México (traducido del italiano por José Joaquín de Mora). Londres: R. Ackermann.

Galindo, J. \& Paredes, J. (2008): Puentes de arco de ladrillo en la región del alto Cauca. Una tradición constructiva olvidada. Bogotá: Universidad Nacional de Colombia.

Garcilaso de la Vega, F. (1984): Comentarios reales. México: Porrúa.

Obregón, D. (1992): Sociedades científicas en Colombia. Bogotá: Banco de la República.

Picón, A. (1988): Architectes et ingénieurs au siècle des lumiéres. Marsella: Parenthèses.

Poveda, G. (1993): Ingeniería e historia de las técnicas (vols. I y II). Bogotá: Colciencias.

Safford, F. (1976): The ideal of the Practical. Colombia's Struggle to Form a Technical Elite. Austin: University of Texas.

Squier, E.G. ( 1877): Peru: Incidents of Travel and Exploration in the Land of the Incas. New York: Harper \& Brothers.

Vérin, H. (1993): La gloire des ingénieurs. L'intelligence technique du XVI au XVII siécle. París: Albin Michel. 